\title{
Erratum to: The characteristics of intrinsic complex micro-contractile activity in isolated strips of the rat bladder
}

\author{
J. I. Gillespie ${ }^{1} \cdot$ C. Rouget ${ }^{2} \cdot$ S. Palea ${ }^{2} \cdot$ C. Granato ${ }^{2,3} \cdot$ L. Birder ${ }^{4} \cdot$ C. Korstanje $^{3}$
}

Published online: 7 August 2015

(C) Springer-Verlag Berlin Heidelberg 2015

\section{Erratum to: Naunyn-Schmiedeberg's Arch Pharmacol (2015) 388:709-718 \\ DOI 10.1007/s00210-015-1131-4}

The original publication of this paper contains error. An incorrect Abstract Section was presented. Below, the correct Abstract Section:

\begin{abstract}
In the resting and un-stimulated state, the bladder wall is not quiescent and discrete contractile events, microcontractions, can be recorded in almost all species. This activity contributes to the active element of compliance and to the basal resting tension. This intrinsic activity underpins the more complex phasic activity, non-voiding activity (NVA) that can be seen to increase progressively as the bladder is filled. The NVA represents the motor component of a motor sensory system that relays information to the CNS on bladder volume. Despite the importance of this intrinsic motor activity, little is known about the mechanisms involved in its
\end{abstract}

The online version of the original article can be found at http://dx.doi.org/ 10.1007/s00210-015-1131-4.

J. I. Gillespie

j.i.gillespie@ncl.ac.uk

1 Uro-physiology Research Group, The Dental and Medical School, Newcastle University, Newcastle upon Tyne NE2 4HH, England, UK

2 UROsphere S.A.S., Faculté des Sciences Pharmaceutiques, 35, Chemin des Maraîchers, 31062 Toulouse Cedex 09, France

3 Astellas Pharma Europe B.V., Sylviusweg 62, 2333 BE Leiden, The Netherlands

4 Department of Medicine, University of Pittsburgh School of Medicine, Pittsburgh, PA, USA generation and modulation. The present experiments were done on isolated hemi-bladders from normal rats and measurements made of the intrinsic motor activity. Detailed analysis of the resting state reveals the presence of discrete phasic contractile events, micro-contractions that range in amplitude from $0.1-0.6 \mathrm{mN}$. These events seem to occur randomly and the basal activity has the appearance of 'noise'. An analysis of the frequency amplitude distribution of the contractile events, reveals that the total activity appears to be the sum of a number of discrete contractile units, each generating a phasic contraction about a specific mean value and with characteristic frequency. In a hemi-bladder, there are between 20-30 units generating the activity at rest. Using the timed integral of the activity (product of amplitude and frequency), it was noted that the activity was increased by the muscarinic agonist carbachol, but it was decreased by the $\beta$-adrenergic agonist isoprenaline. Stretching the preparations also increased the activity. Using these observations, a simple model of the structural and functional organisation of the isolated rat bladder is proposed: the wall appears to be arranged into a number of discrete motor units acting independently. However, the activity can be stimulated or inhibited by pharmacological agents and mechanically (stretch). The possible relevance of this activity, its relationship to NVA and in relation to the mode of action of drugs are discussed. 